\title{
Automatic Fast Moving Object Detection in Video of Surveillance System
}

\author{
Pranali A. Pojage ${ }^{1}$, Ajay A. Gurjar ${ }^{2}$ \\ ME Scholar, Electronics \&Telecommunication Engineering, Sipna College of Engineering \& Technology, Amravati, \\ Maharashtra, India ${ }^{1}$ \\ Professor, Electronics \&Telecommunication Engineering, Sipna College of Engineering \& Technology, Amravati, \\ Maharashtra, India ${ }^{2}$
}

\begin{abstract}
Moving object detection is the task of identifying the physical movement of an object in a given region or area. Over last few years, moving object detection has received much of attraction due to its wide range of applications like video surveillance, human motion analysis, robot navigation, event detection, video conferencing, traffic analysis and security. In addition, moving object detection is very consequential and efficacious research topic in field of computer vision and video processing, since it forms a critical step for many complex processes like video object classification and video tracking activity. Consequently, identification of actual shape of moving object from a given sequence of video frames becomes pertinent. However, task of detecting actual shape of object in motion becomes tricky due to various challenges like dynamic scene changes, illumination variations, and presence of shadow, camouflage and bootstrapping problem. To reduce the effect of these problems, researchers have proposed number of new approaches. This project provides a brief classification of the classical approaches for moving object detection.
\end{abstract}

Keywords: Moving Object Detection, Object Classification, Video Surveillance, Video Frames.

\section{INTRODUCTION}

A video is a group of basic structural units, such as scene, shot and frame associated with audio data. A frame is defined as a single picture shot of movie camera, led by many successive frames for seamless video. Moving object detection is the act of segmenting non-stationary objects of interest with respect to surrounding area or region from a given sequence of video frames. Determination of the moving target forms the basic step for classification and tracking process of object in motion. The main aim of moving object detection and tracking activity is to discover foreground moving target either in every video frame or at very first appearance of moving target in video. In any video analysis activity there are three major phases: identification of the moving target (object) and analysis of the moving target (object) in order to determine its behaviour. Hence, identifying the moving object becomes significant step for any analysis process.

Moving object detection has become a central topic of discussion in field of computer vision due to its wide range of applications like video surveillance, monitoring of security at airport, law enforcement, video compression, automatic target identification, marine surveillance and human activity recognition. Several methods have been proposed so forth for object detection, out of which Background Subtraction, Frame differencing, Temporal Differencing and Optical Flow are extensively used traditional methods.

Moving object detection has always proved to be challenging task due to number of factors like dynamic background, illumination variations, and misclassification of shadow as object, camouflage and bootstrapping problems.

\section{LITERATURE REVIEW}

From the rigorous review of related work and published literature it is observed that many researchers have designed different techniques.

Dong et al. presents new approach based on RGB color space along with edge ratio that allows determining moving object and shadow separately. It is achieved in three consecutive steps. Initially, specific characteristics of moving target and shadow are analyzed in three dimensional RGB color space. Subsequently, object and shadow are differentiated based on the chromaticity and brightness distortion of the pixels of current image and background image. Ultimately, misclassified object and shadow are treated by area and edge ratio of each region. Separation of object, shadow and background using RGB color space model considering chromaticity and brightness ratio model combined with edge ratio model for treatment of misclassified object and shadow. Moving object and shadow are determined 
separately. Fast enough for utilization in real time analysis. Darker shadow areas or moving target having similar color information to that of background area will lead to failure [1].

Choi et al. introduces a novel approach for moving object identification under fast illumination changing condition. The proposed approach is depended on chromaticity model and brightness ratio model. The main focus of the proposed approach is to eliminate false foreground pixels detected by Gaussian mixture model under fast illumination variations. At the outset, probability distribution of false foreground pixels is determined by chromaticity difference model in order to separate pixels as foreground pixels of moving target and candidate false foreground pixels. However it is possible that candidate false foreground pixel may contain moving target pixel because of zero chromaticity difference. These pixels are then separated by brightness ratio model. As a result of which pixel indicating the actual moving target are identified with elimination false foreground pixels. Identification of moving target under fast illumination variations using Gaussian mixture model for object detection and chromaticity and brightness ration model for elimination of false foreground pixels. Does not require training sequence. Automatic adjustment of the parameters. Results degrade in complex environment that has piled snow, puddles or in seculars regions [2].

Hao et al. presented a fast and robust approach originated from combined spatio-temporal background and foreground modeling. In order to adapt changes with background in each video frame, prior probabilities are estimated. Firstly, by making use of kernel density estimation; temporal and spatial information are obtained for background modeling. Secondly, to develop foreground model, Gaussian formulation is used to depict the spatial correlation between targets in motion. Lastly, a fusion background frame is produced along with proposal of updating rates. Approach for traffic surveillance using Bayesian fusion method where in kernel density estimation is used for background modeling and Gaussian formulation is carried out for foreground model. Requires less computational time. Works well with rapidly and slowly changing background Object's feature identical to that of background are abolished [3].

Gang et al proposed an enhanced version of traditional three-frame differential method. Where in Canny edge detection algorithm is being combined along with three frame differential approach to obtain more complete information regarding moving object. Firstly, by making using of canny edge detector and noise removal technique, reasonably clear boundary image of object is obtained. Secondly, dilation operation is applied in which background points are merged with object. Secondly, local boundary connection is applied to gain the clarity of boundary of moving object. In final stage of the proposed method, black and white connected domain area of moving target and background is converted into binary form. Enhanced three frame differential method combined with canny edge detection to gain complete information related to moving target. Ghosting effect is eliminated; Algorithm beats the empty phenomenon and edge deletion problems of standard three-frame differential method. The result is not ideal in the environment with strong light and obvious shadow. Results degrade for dynamic background [4].

\section{III.PROPOSED WORK}

A. Classification of traditional approaches of moving object detection.

Traditional Approaches for moving object detection can be broadly categorized into four forms as Background Subtraction, Frame Differencing, Temporal Differencing and Optical Flow. Fig. 1 shows a brief classification of traditional approaches.

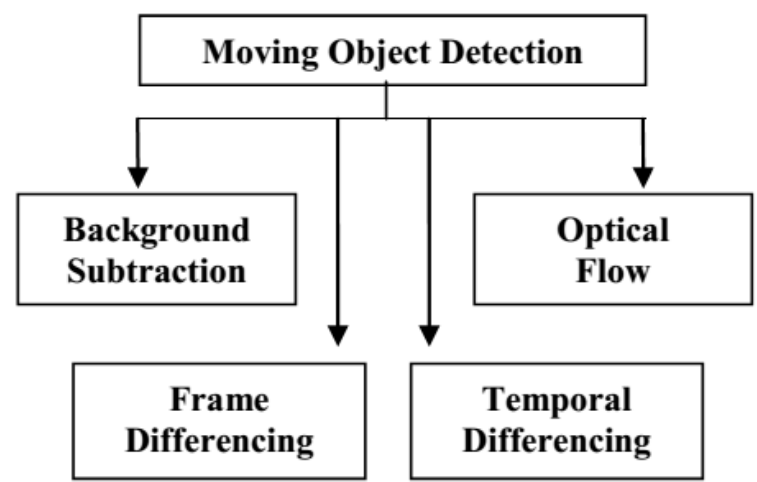

Fig 1. Traditional Approaches of Moving Object Detection

1. Background Subtraction

Background Subtraction Method is considered to be one of the most reliable method for moving object detection. Background subtraction works by initializing a background model, then difference between current frame and 
presumed background model is obtained by comparing each pixel of the current frame with assumed background model color map. In case difference between colors is more than threshold, pixel is considered to be belonging to foreground. Performance of traditional background subtraction method mainly gets affected when background is dynamic, illumination changes or in presence of shadow.

Numerous methods have been developed so forth to upgrade background subtraction method and overcome its drawbacks. Different methods of background subtraction as reviewed by Massimo Piccardi are: Concurrence of image variations, Eigen backgrounds, Mixture of Gaussians, Kernel density estimation (KDE), Running Gaussian average, Sequential KD approximation and Temporal median filter.

\section{Frame Differencing}

Frame difference method identifies the presence of moving object by considering the difference between two consecutive frames. The traditional approach makes use of image subtraction operator that obtains output image by subtracting second image frame from first image frame in corresponding consecutive frames. Frame differencing method lacks in obtaining the complete contour of the object as a result of which morphology operations are general used to obtain better results.

\section{Temporal Differencing}

Temporal differencing method detects the moving target by employing pixel-wise difference method among two successive frames. Traditional temporal difference method is flexible to dynamic changes in the scenes but results degrade when moving target moves slowly since due to minor difference between consecutive frames, object is lost. Moreover, trailing regions are detected wrongly as moving object (ghost region) because of fast movement of object, additionally incorrect detection will result where objects preserve uniform regions.

\section{Optical Flow}

Optical flow approach of moving target detection is based on calculation of optical flow field of image (or video frame). Clustering is performed on the basis of the obtained optical flow distribution information obtained from the image (video frame). This method allows obtaining complete knowledge about the movement of the object and is useful to determine moving target from the background. However, this method suffers from some of drawbacks like large quantity of calculations are required to obtain optical flow information and it is sensitivity to noise.

\section{METHODOLOGY}

First we are taking video which is input to the system. Then frame is extracted from video sequence, it is assumed that camera is stationary and there is no change in background. After that we are taking the difference of two frames, frame differencing is applied for detecting the existence and position of a moving objects. Each extracted sub image is subtracted from the respective portion of a ground imageto determine the existence of an object.

Frame difference method identifies the presence of moving object by considering the difference between two consecutive frames. The traditional approach makes use of image subtraction operator that obtains output image by subtracting second image frame from first image frame in corresponding consecutive frames. Frame differencing method lacks in obtaining the complete contour of the object as a result of which morphology operations are general used to obtain better results.

After that identify the objects which are changing its position in a successive frames. We can also define position of objects in which it is moving, that is from left to right or from up to down and vice versa. The object position in each frame is noted and store in $\mathrm{X}$ and $\mathrm{Y}$ array. The difference of consecutive $\mathrm{X}$ and $\mathrm{Y}$ position give us the movement direction as follows.

$$
\begin{aligned}
X \_ \text {diff } & =X(\mathrm{k})-\mathrm{X}(\mathrm{k}+1) \\
\mathrm{Y} \_ \text {diff } & =\mathrm{Y}(\mathrm{k})-\mathrm{Y}(\mathrm{k}+1)
\end{aligned}
$$

If the $\mathrm{X}$ _diff is positive and the difference is more as compare to $\mathrm{Y}$ _diff, which is slightly varying or constant. We conclude that object is moving from left to right. Similarly If the $X_{-}$diff is negative and the difference is more as compare to Y_diff, which is slightly varying or constant. We conclude that object is moving from right to left.

If the Y_diff is positive and the difference is more as compare to X_diff, which is slightly varying or constant. We conclude that object is moving from down to up. Similarly If the $Y_{-}$diff is negative and the difference is more as compare to X_diff, which is slightly varying or constant. We conclude that object is moving from up to down. 


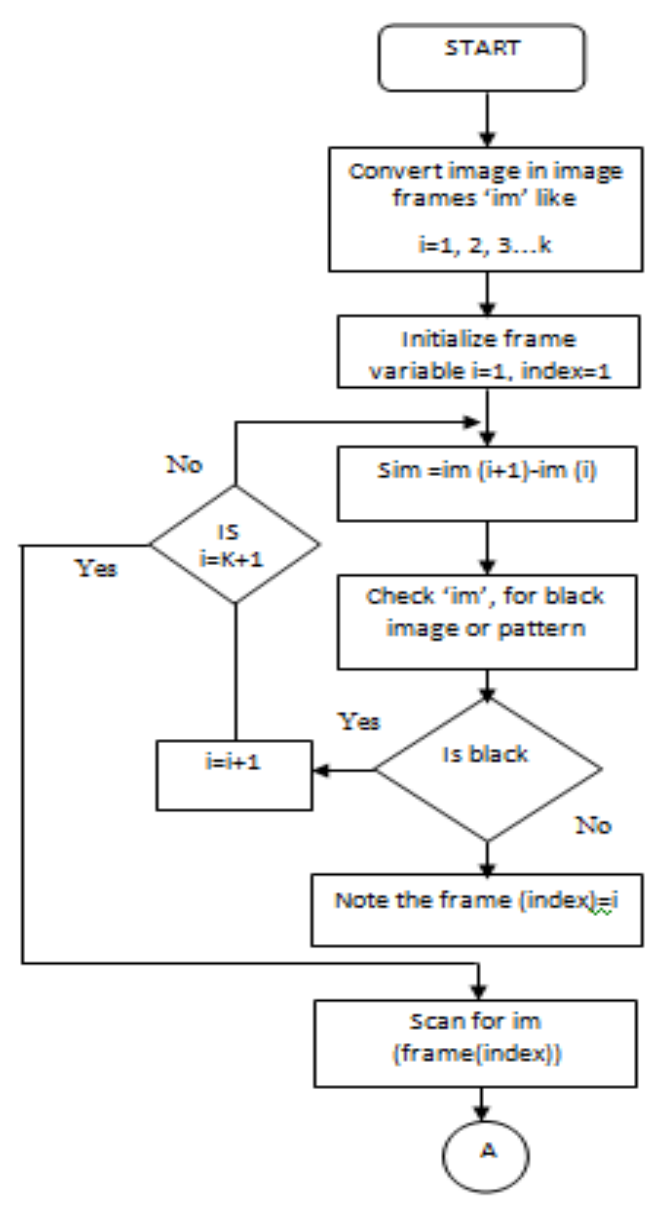

Fig. 2 Flowchart of Methodology (a)

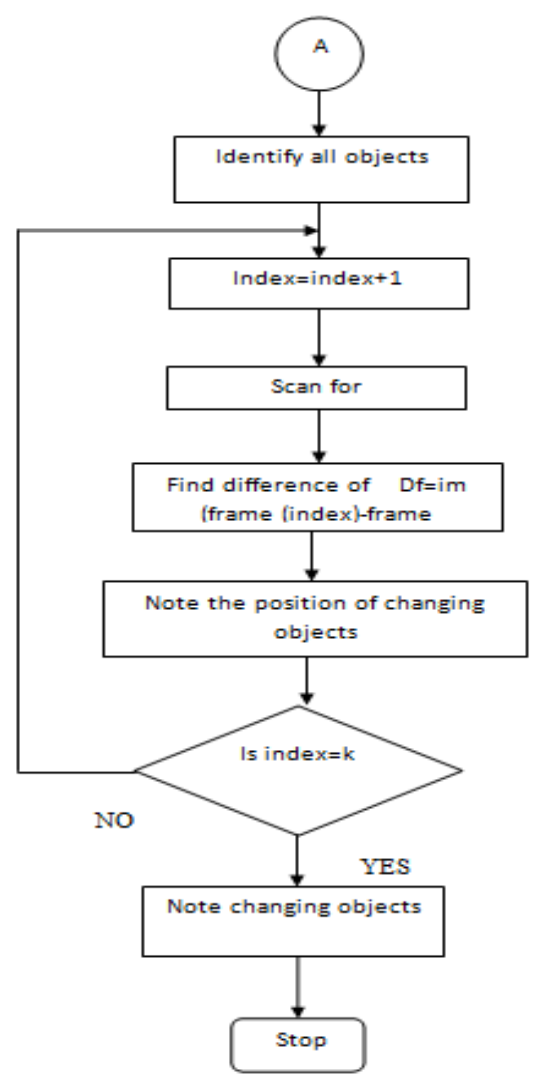

Fig 3. Fig. 1 Flowchart of Methodology (b) 


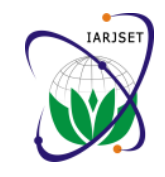

If $\mathrm{X}$ _diff is positive and more as well $\mathrm{Y}$ _diff is positive and more, we conclude that the object is moving from bottomleft to top-right.If $\mathrm{X}$ _diff is negative and more as well $\mathrm{Y}$ _diff is positive and more, we conclude that the object is moving from bottom-right to top-left. If $\mathrm{X}$ _diff is positive and more as well $\mathrm{Y}$ _diff is negative and more, we conclude that the object is moving from top-left to bottom-right.If $\mathrm{X}$ _diff is negative and more as well Y_diff is negative and more, we conclude that the object is moving from top-right to bottom-left.

\section{RESULT}

1. Output when object is moving from right to left

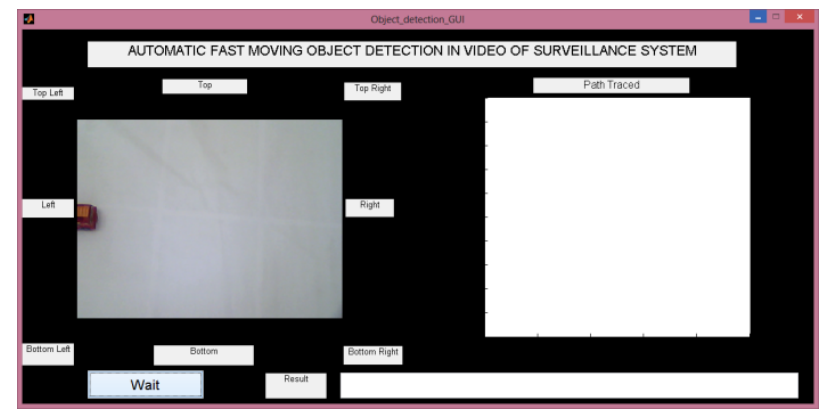

Fig4: Object is moving from right to left

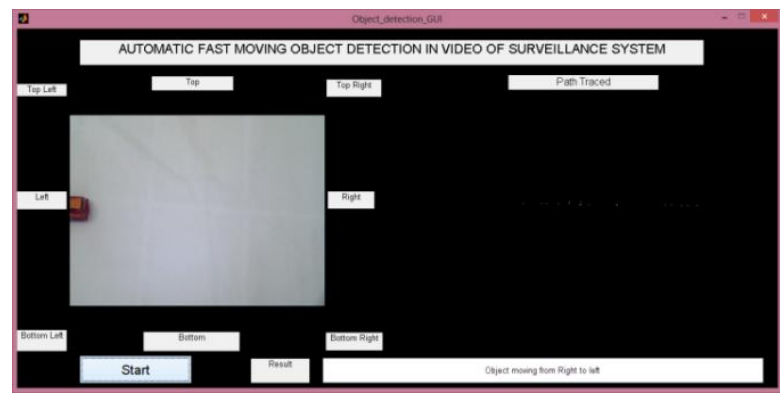

Fig5: Path of object when it is moved from right to left

2. Output when object is moving from bottom to top

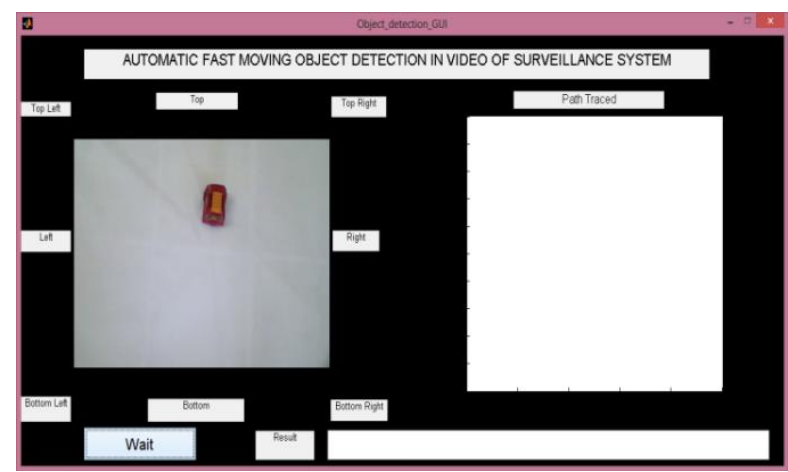

Fig6: Object is moving from bottom to top

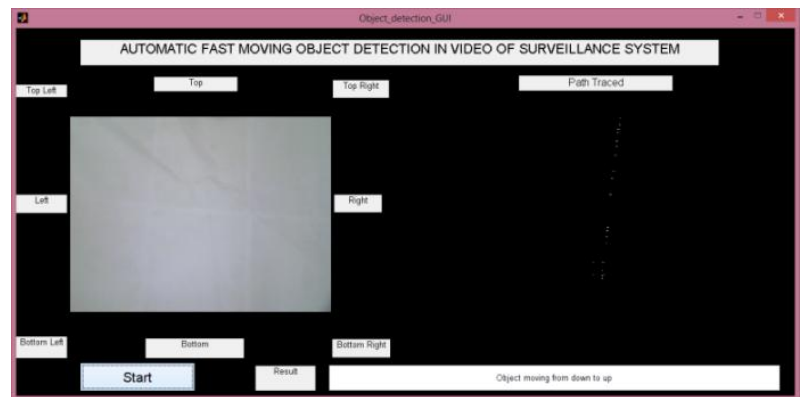

Fig7: Path when object is moving from bottom to top 


\section{IARJSET

3. Output when object is moving from top right to bottom left

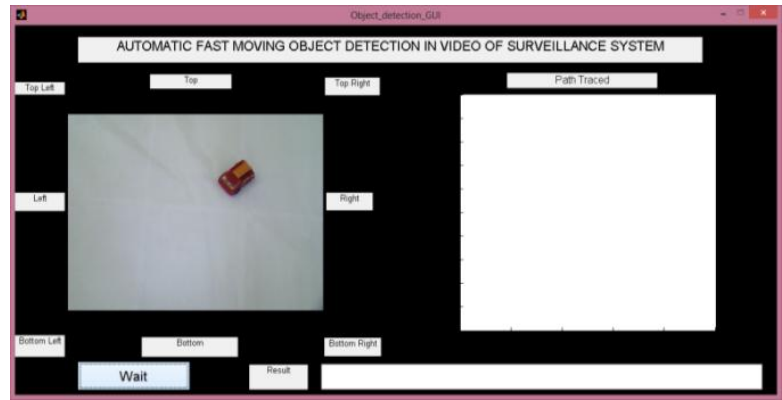

Fig8: Object is moving from top right to bottom left

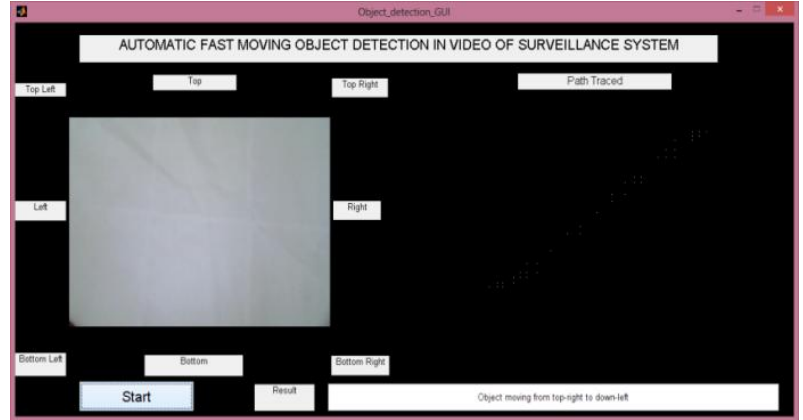

Fig9: Path when object is moving from top right to bottom left

\section{CONCLUSION}

Moving Object Detection is very momentous and efficient research field that is powerfully motivated by number of applications. The Project is to present an outline of established tactics for moving object detection and study of recent growth in corresponding theme is depicted with the focus on the shortcomings of conventional method.

Temporal information based methods like background subtraction; frame difference and temporal difference were witnessed as chiefly used approaches for determination of object in motion for video sequence recorded using stationary single camera. During survey it was identified that shadow, illumination variation and dynamic background are the major problems which are worked over since these problems lead to reduction in the accuracy of successive steps of analysis process i.e., classification and tracking. Abundant of work has been done so forth to deal with shadow and illumination variation using background subtraction model. Though the enhanced work provides better result compared to conventional approaches, but require more computational time and need additional algorithm to deal with complex environment. Improvisation in frame difference method allows to obtain entire contour of the object with comparatively less computational time than background subtraction model but it is vulnerable to dynamic changes and yet no well established method specific to frame difference for shadow elimination and illumination variation is developed. Advancement in temporal difference method determines entire contour of object with dynamic changes, but still alike frame difference method, no particular method for shadow purging and changes in lighting conditions for temporal difference method was recognized.

\section{REFERENCES}

[1] J. Breckling, Ed Xia Dong, Kedian Wang and Guohua Jia, "Moving Object and Shadow Detection Based on RGB Color Space and Edge Ratio," IEEE $2^{\text {nd }}$ International Conference, on Image and Signal Processing, pp. 1-5, Oct. 2009.

[2] JinMin Choi, Hyung Jin Chang, Yung Jun Yoo and Jin Young Choi, "Robust moving object detection against fast illumination change," Computer Vision and Image Understanding, pp. 179-193, 2012.

[3] JiuYue Hao, Chao Li, Zuwhan Kim, and Zhang Xiong. "SpatioTemporal Traffic Scene Modeling for Object Motion Detection," IEEE, Intelligent Transportation Systems, 2012.

[4] Liu Gangl, Ning Shangkun ,You Yugan ,Wen Guanglei and Zheng Siguo, "An Improved Moving Objects Detection Algorithm,” in Proceedings of the 2013 IEEE International Conference on Wavelet Analysis and Pattern Recognition, pp. 96-102, 14-17 July, 2013.

[5] Huijuan Zhang and Hanmei Zhang, "A Moving Target Detection Algorithm Based on Dynamic Scenes," IEEE Conference on Computer Science \& Education, pp. 995-998, April 2013.

[6] Lucia Maddalena and Alfredo Petrosino, "The 3dSOBS+ algorithm for moving object detection," Computer Vision and Image Understanding, pp. 65-73, 2014.

[7] Prem Kumar Bhaskar and Suet-Peng Yong, "Image Processing Based Vehicle Detection and Tracking Method," IEEE, 2014.

[8] Zhihu Wang, Kai Liao, Jiulong Xiong, and Qi Zhang, "Moving Object Detection Based on Temporal Information," IEEE Signal Processing Letters, vol. 21, no. 11, pp. 1404-1407, November 2014.

[9] Jinhai Xiang, Heng Fan, Honghong Liao,Jun Xu,Weiping Sun and Shengsheng Yu, "Moving Object Detection and Shadow Removing under Changing Illumination Condition,” Hindawi Publishing Corporation, Mathematical Problems in Engineering, pp. 1-10, February 2014. 